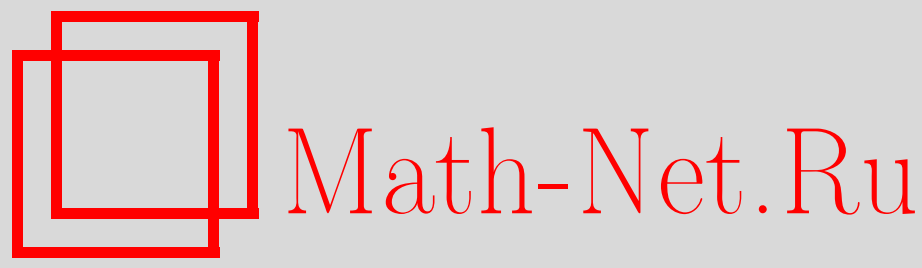

А. В. Маршаков, Интегралы периодов, квантовые числа и конфайнмент в суперсимметричной КХД, ТМФ, 2010, том 165, номер 3, 488-502

DOI: https://doi.org/10.4213/tmf6589

Использование Общероссийского математического портала Math-Net.Ru подразумевает, что вы прочитали и согласны с пользовательским соглашением http://www . mathnet.ru/rus/agreement

Параметры загрузки:

IP : 18.208 .226 .222

26 апреля 2023 г., $17: 25: 05$

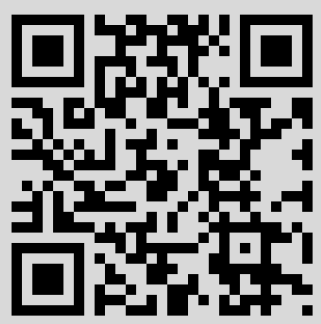




\title{
ИНТЕГРАЛЫ ПЕРИОДОВ, КВАНТОВЫЕ ЧИСЛА И КОНФАЙНМЕНТ В СУПЕРСИММЕТРИЧНОЙ КХД
}

\begin{abstract}
Приведено явное вычисление интегралов периодов на вырожденных кривых Виттена-Зайберга для суперсимметричной КХД, а также показано, как именно эти периоды определяют изменение квантовых чисел состояний при переходе из области слабой связи в район сильной связи в пространстве модулей масс теории. Обсуждается конфайнмент монополей в сильной связи, демонстрируется, что неоднозначность в выборе пути в пространстве модулей не влияет на физические выводы о конфайнменте монополей в фазе с конденсированными легкими дионами.
\end{abstract}

Ключевые слова: суперсимметричные калибровочные теории, конфайнмент, римановы поверхности, интегрируемые системы.

\section{1. ВВЕДЕНИЕ}

Суперсимметричная КХД в последнее время играет роль лаборатории по исследованию конфайнмента. Не будучи достаточно реалистичной теорией, чтобы описывать окружающий мир, она тем не менее может рассматриваться как модель квантовой теории, доступная непертурбативному анализу, и поэтому может существенно продвинуть горизонт нашего понимания, по крайней мере в принципе, того, что может происходить в калибровочной теории в сильной связи.

Ниже представлены некоторые подробности изучения свойств конфайнмента в суперсимметричной КХД в соответствии с программой работ [1], [2], а также завершено обсуждение ряда технических тонкостей, возникающих при этом в лежащей в основе комплексной геометрии. Основная идея рассматриваемого сценария заключается в том, чтобы начать с очевидного конфайнмента монополей типа (дуального) эффекта Мейсснера в кварковом вакууме суперсимметричной калибровочной теории в слабой связи [1], а затем продвигать эту картину, меняя массовые параметры теории, в область сильной связи [2]. Таким путем мы приходим к эффективной теории легких дионов, заменяющих изначальные кварки, так как БПС-состояния

\footnotetext{
*Физический институт им. П. Н. Лебедева РАН, Москва, Россия. E-mail: mars@lpi.ru

${ }^{\dagger}$ Институт теоретической и экспериментальной физики, Москва, Россия. E-mail: mars@itep.ru
} 


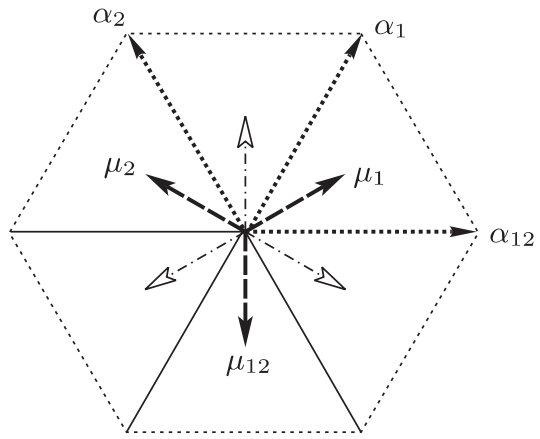

Рис. 1. Корни $\boldsymbol{\alpha}$ и фундаментальные веса $\boldsymbol{\mu}$ для алгебры Ли калибровочной группы $S U(3)$ в картановской плоскости, корни нормированы канонически условиями $\boldsymbol{\alpha}^{2}=2$. Обозначения для корней выбраны в соответствии с условием ортогональности $\boldsymbol{\mu}_{i} \cdot \boldsymbol{\alpha}_{j}=\delta_{i j}, i, j=1,2$, с весами $\boldsymbol{\mu}_{1}$ и $\boldsymbol{\mu}_{2}$ фундаментального представления $\mathbf{3}$ (веса двойственного фундаментального представления $\overline{\mathbf{3}}$ изображены штриховыми линиями). Общее условие дуальности $\boldsymbol{\mu} \cdot \boldsymbol{\alpha} \in \mathbb{Z}$ для произвольных векторов $\boldsymbol{\mu}$ из решетки весов и $\boldsymbol{\alpha}$ из ее подрешетки корней превращается в условие квантования Дирака для (хромо)электрических и магнитных зарядов.

меняют свои квантовые числа из-за нетривиальных монодромий в пространстве модулей масс. Исследование этих монодромий является нетривиальной задачей (см., например, работы [3], [4]) и максимально просто может быть проведено путем вычисления интегралов по периодам и исследования возмущения точек ветвления на (почти) сингулярных кривых Виттена-Зайберга [5]-[8].

Следуя работам [1], [2], мы рассмотрим в качестве базовой модели суперсимметричную КХД с калибровочной группой $S U\left(N_{\mathrm{c}}\right)$ и большим числом $N_{\mathrm{f}}$ фундаментальных ароматов, $N_{\mathrm{c}} \leqslant N_{\mathrm{f}} \leqslant 2 N_{\mathrm{c}}$ : первым нетривиальным (и основным в настоящей статье) примером является $S U(3)$-калибровочная теория с $N_{\mathrm{f}}=4$ или $N_{\mathrm{f}}=5$. Квантовые числа легких состояний можно увидеть на рис. 1, где изображены цветные заряды кварков (фундаментальные веса) и заряды монополей (корни) для калибровочной группы $S U(3)$. В контексте теории Виттена-Зайберга [5], [6], которая необходима для изучения точных свойств суперсимметричной КХД в окрестности вакуумов в области сильной связи, условие квантования Дирака $\boldsymbol{\mu}_{i} \cdot \boldsymbol{\alpha}_{j}=\delta_{i j}$ принимает вид формы пересечений циклов $A_{i} \circ B_{j}=\delta_{i j}$ на спектральной кривой (см. рис. 2 для калибровочной группы $S U(3))$. В дальнейшем мы будем пользоваться только "гомологической нормировкой" зарядов [6], в которой они измеряются в циклах на кривой Виттена-Зайберга, и поэтому всегда целочислены.

Преобразования дуальности не меняют комплексной структуры на кривых Виттена-Зайберга, но переставляют электрические $A$-циклы с магнитными $B$-циклами, и поэтому отвечают электромагнитной дуальности [5], [6]. Важно, что периоды

$$
a_{i}=\frac{1}{2 \pi i} \oint_{A_{i}} d S, \quad a_{i}^{\mathrm{D}}=\frac{1}{2 \pi i} \oint_{B_{i}} d S,
$$




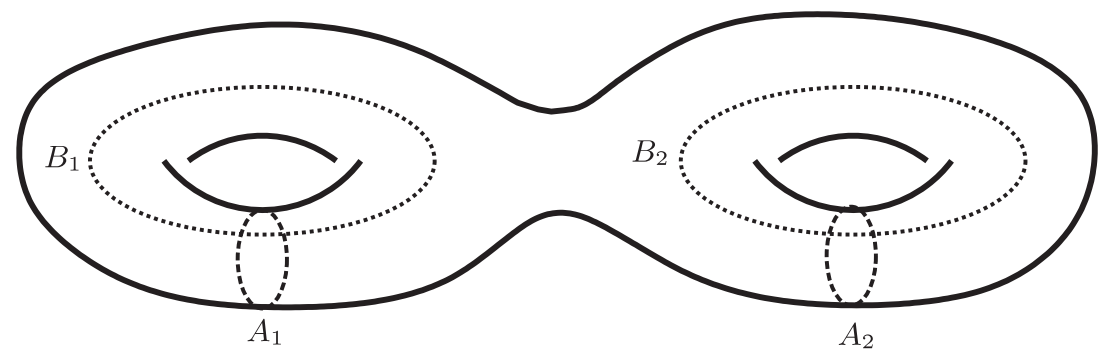

Рис. 2. $A$ - и $B$-циклы и их форма пересечений $A_{i} \circ B_{j}=\delta_{i j}$ римановой поверхности рода $N_{\mathrm{c}}-1=2$ в случае $S U(3)$-калибровочной теории. Элементарные заряды кварков $\boldsymbol{\mu}_{1,2} \leftrightarrow A_{1,2}$ отвечают $A$-циклам, в то время как заряды монополей $\boldsymbol{\alpha}_{1,2} \leftrightarrow B_{1,2}-B$-циклам.

входящие (вместе с вычетами $m_{A}=\operatorname{res}_{P_{A}} d S$ ) в массовую формулу БПС-состояний

$$
\operatorname{Mass}_{(\boldsymbol{\mu}, \boldsymbol{\alpha}, \mathbf{b})} \sim\left|\boldsymbol{\mu} \cdot \mathbf{a}+\boldsymbol{\alpha} \cdot \mathbf{a}^{\mathrm{D}}+b_{A} m_{A}\right|,
$$

никогда не бывают вещественными одновременно, за исключением сингулярных или вырожденных случаев, когда некоторые из этих периодов обращаются в нуль, в результате в спектре появляются дополнительные безмассовые состояния. Безмассовость этих состояний приводит к дополнительной возможности распадов, изменяющих квантовые числа легких состояний, а поэтому в различных областях пространства модулей в конденсаты выпадают различные заряды. Чтобы определить эти заряды, следует воспользоваться теорией Виттена-Зайберга для суперсимметричной КХД в окрестности сингулярных кривых, отвечающих $\mathcal{N}=1$ вакуумам.

\section{2. ТЕОРИЯ ВИТТЕНА-ЗАЙБЕРГА ДЛЯ СУПЕРСИММЕТРИЧНОЙ КХД}

Кривая общего вида для $\mathcal{N}=2$ суперсимметричной $\mathrm{KXД} \mathrm{с} N_{\text {с }}$ цветами и $N_{\mathrm{f}}$ ароматами может быть записана в виде [7], [9]

$$
y^{2}=P^{2}(x)-4 Q(x),
$$

где

$$
\begin{aligned}
& P(x)=\prod_{i=1}^{N_{\mathrm{c}}}\left(x-\phi_{i}\right), \quad \sum_{i=1}^{N_{\mathrm{c}}} \phi_{i}=-\Lambda \delta_{N_{\mathrm{f}}, 2 N_{\mathrm{c}}-1}, \\
& Q(x)=\Lambda^{2 N_{\mathrm{c}}-N_{\mathrm{f}}} \prod_{A=1}^{N_{\mathrm{f}}}\left(x+m_{A}\right)
\end{aligned}
$$

являются полиномами степеней $N_{\text {с }}$ и $N_{\mathrm{f}}$ соответственно. В квазиклассическом режиме корни $\phi_{i}, i=1, \ldots, N_{\mathrm{c}}$, полинома $P(x)$ совпадают с собственными значениями матрицы $\Phi$ конденсата комплексного скаляра из векторного мультиплета $\mathcal{N}=2$ суперсимметричной теории Янга-Миллса, но, будучи вычисленными точно, они (точнее, симметрические функции от них) приобретают (зависящие от $\Lambda$ и $m_{A}$ ) поправки благодаря инстантонным эффектам. 
Гиперэллиптическое представление (3) может быть также переписано в виде

$$
w+\frac{Q(x)}{w}=P(x)
$$

или в виде $\mathrm{e}^{1)}$

$$
W+\frac{1}{W}=\frac{P(x)}{\sqrt{Q(x)}},
$$

где $y=w-Q(x) / w=(W-1 / W) \sqrt{Q(x)}$.

Кривые (3), (5) или (6) оснащены производящим дифференциалом

$$
d S \sim x \frac{d w}{w}=x \frac{d W}{W}+\frac{1}{2} x \frac{d Q}{Q}=\frac{x d P}{y}-x \frac{P}{2 y} \frac{d Q}{Q}+\frac{1}{2} x \frac{d Q}{Q},
$$

периоды которого (1) входят в массовую формулу (2) одновременно с его вычетами

$$
\operatorname{res}_{P_{A}^{ \pm}} d S=\left.m_{A} \cdot \frac{P}{2 y}\right|_{x=-m_{A}}-\frac{m_{A}}{2}=-m_{A}
$$

в точках $P_{A}^{ \pm}$, где $x\left(P_{A}^{ \pm}\right)=-m_{A}$ на одном из листов кривой (3). Вычеты (8) должны обращаться в нуль в безмассовом пределе [6], и при $N_{\mathrm{f}}=2 N_{\mathrm{c}}-1$ это требование приводит к появлению ненулевого члена порядка $\Lambda$ в правой части второго равенства в формуле (4). Вариация дифференциала (7) при постоянном $W$ приводит к формуле

$$
\delta d S \sim \frac{d x}{y}\left(\delta P(x)-\frac{1}{2} P \frac{\delta Q(x)}{Q(x)}\right) .
$$

В случае калибровочной группы $S U(3)$ при $N_{\mathrm{f}}<5$ и $\phi_{3}=-\phi_{1}-\phi_{2}$ удобно ввести переменные $u, v$ с помощью равенства

$$
P(x)=\left(x-\phi_{1}\right)\left(x-\phi_{2}\right)\left(x-\phi_{3}\right)=x^{3}-u x-v,
$$

так что

$$
u=\phi_{1}^{2}+\phi_{2}^{2}+\phi_{1} \phi_{2}, \quad v=-\phi_{1} \phi_{2}\left(\phi_{1}+\phi_{2}\right) .
$$

Теперь мы рассмотрим частные случаи этих формул в окрестности сингулярных кривых.

\section{3. ИНТЕГРАЛЫ ПЕРИОДОВ ДЛЯ ВЫРОЖДЕННЫХ КРИВЫХ}

3.1. Случай $N_{\mathrm{c}}=2$ и $N_{\mathrm{f}}=2$. Начав с $S U(3)$-калибровочной теории в окрестности вакуума с двумя конденсированными ароматами $(r=2$ в терминах работы [1]) в области слабой связи и двигаясь в область сильной связи, мы получим картину,

\footnotetext{
1) Иногда (например, с точки зрения бранных конструкций [10] и возможной связи с матричными моделями [11] и квантовыми интегрируемыми системами) более адекватным является асимметричное представление с факторизованным $Q(x)=Q_{+}(x) Q_{-}(x)$ и $w=\mathrm{w} Q_{+}(x)$, так что уравнение (5) принимает вид $\mathrm{w}^{2} Q_{+}(x)-P(x) \mathrm{w}+Q_{-}(x)=0$.
} 
эффективно описываемую $S U(2)$-калибровочной теорией с $N_{\mathrm{f}}=2$ легкими ароматами, когда $r=2$ вакуум сталкивается с вакуумом, содержащим безмассовые монополи [2]. В этом полезном примере теории с $N_{\mathrm{c}}=2$ и $N_{\mathrm{f}}=2$, взятыми для простоты с совпадающими массами $m_{1}=m_{2}=m$, для кривой (3) получаем

$$
y^{2}=\left(x^{2}-u\right)^{2}-4 \Lambda^{2}\left(x+m_{1}\right)\left(x+m_{2}\right)=\left(x^{2}-u\right)^{2}-4 \Lambda^{2}(x+m)^{2} .
$$

Производящий дифференциал (7) превращается при совпадающих массах в

$$
\begin{gathered}
d S \sim \frac{x d P}{y}-x \frac{P}{2 y} \frac{d Q}{Q}+\frac{1}{2} x \frac{d Q}{Q}=\frac{x d P}{y}-x \frac{P}{y} \frac{d q}{q}+x \frac{d q}{q}, \\
q(x)=\prod_{B=1}^{N_{\mathrm{f}} / 2}\left(x+m_{B}\right),
\end{gathered}
$$

где мы выбрали $m_{B+N_{\mathrm{f}} / 2}=m_{B}, B=1, \ldots, N_{\mathrm{f}} / 2$, для четного числа ароматов $N_{\mathrm{f}}$. Кривая (11) отвечает $P(x)=x^{2}-u$ и $q(x)=x+m$.

В кварковом вакууме, т. е. в точности в точке $u=u_{\mathrm{Q}}=m^{2}$, кривая (11) еще более вырождается и задается уравнением

$$
y^{2}=(x+m)^{2}\left((x-m)^{2}-4 \Lambda^{2}\right) \equiv(x+m)^{2} Y^{2},
$$

где $Y^{2}=(x-m)^{2}-4 \Lambda^{2}$, а дифференциал Виттена-Зайберга (7) принимает вид

$$
d S=\frac{x d x}{Y}+\frac{x d x}{x+m}
$$

где первое слагаемое в правой части равенства совпадает с производящим дифференциалом формальной чистой $\mathcal{N}=2$ суперсимметричной $U(1)$-калибровочной теории с единственным вакуумным средним, равным $m$ [12], [13].

Благодаря условию (8) и тому факту, что на вырожденной кривой положение массового полюса в $x=-m$ (для обоих ароматов) совпадает с вырожденным разрезом, дифференциал (14) нормирован следующим образом:

$$
\begin{aligned}
& \frac{1}{2 \pi i} \oint_{x=-m} d S_{+}=\frac{1}{2 \pi i} \oint_{A^{+}} d S_{+}=a=-m, \\
& \frac{1}{2 \pi i} \oint_{x=-m} d S_{-}=\operatorname{res}_{x=-m} d S_{-}-\frac{1}{2 \pi i} \oint_{A^{-}} d S_{-}=-2 m+a=-m,
\end{aligned}
$$

что, очевидно, выполняется для (14). Так как кривая (13) рациональна, дифференциал (14) может быть явно проинтегрирован:

$$
S=Y+m \ln (x-m+Y)+x-m \ln (x+m) .
$$

Для того чтобы вычислить нужный $B$-период (массу монополя), следует взять разность $\left.S_{+}\right|_{x=-m}-\left.S_{-}\right|_{x=-m}$ значений интеграла (16) на двух различных листах римановой поверхности (13). Это невозможно сделать путем прямой подстановки 
значения $x=-m$ в формулу (16) из-за логарифмической особенности, т.е. кривая (13) является “слишком вырожденной" 2). Слегка регуляризируем ее и обозначим расстояние между положением полюса и ближайшим к нему концом разреза через $\epsilon^{ \pm}$в зависимости от листа $Y=Y_{ \pm}$кривой (13). Величины $\epsilon^{ \pm}=\epsilon^{ \pm}(m, \Lambda)$ можно определить следующим образом (см., например, работы [13]): у дифференциала

$$
d \phi=\frac{d w}{w} \stackrel{(13)}{=} \frac{d x}{Y}+\frac{d x}{x+m}
$$

периоды должны быть константами [16], более того, его $B$-периоды на кривой (13) можно выбрать просто равными нулю. Интегрируя (17), получаем

$$
\phi=\ln (x-m+Y)+\ln (x+m)
$$

полагая $\left.\phi_{+}\right|_{x=-m}-\left.\left.\phi_{-}\right|_{x=-m} \equiv \phi_{+}\right|_{x=-m+\epsilon^{+}}-\left.\phi_{-}\right|_{x=-m+\epsilon^{-}}=0$, имеем (при $\left.\epsilon^{ \pm} \rightarrow 0\right)$

$$
\ln \frac{\epsilon^{+}}{\epsilon^{-}}=\ln \frac{m+\sqrt{m^{2}-\Lambda^{2}}}{m-\sqrt{m^{2}-\Lambda^{2}}} .
$$

Поэтому

$$
\begin{aligned}
\left.S_{+}\right|_{x=-m}-\left.S_{-}\right|_{x=-m} & =-m \ln \frac{\epsilon^{+}}{\epsilon^{-}}+\left.2 Y\right|_{x=-m}+m \ln \frac{-2 m+\left.Y\right|_{x=-m}}{-2 m-\left.Y\right|_{x=-m}}= \\
& =4 \sqrt{m^{2}-\Lambda^{2}}+2 m \ln \frac{m-\sqrt{m^{2}-\Lambda^{2}}}{m+\sqrt{m^{2}-\Lambda^{2}}} .
\end{aligned}
$$

Таким образом, вычисление $B$-периода для вырожденной кривой (13) приводит к явной формуле (ср. с работами [17], где похожие структуры возникали опосредованным образом из двумерного подхода)

$$
a^{\mathrm{D}}=\frac{1}{2 \pi i}\left(\left.S_{+}\right|_{x=-m}-\left.S_{-}\right|_{x=-m}\right)=-\frac{i}{\pi}\left(2 \sqrt{m^{2}-\Lambda^{2}}+m \ln \frac{m-\sqrt{m^{2}-\Lambda^{2}}}{m+\sqrt{m^{2}-\Lambda^{2}}}\right),
$$

утверждающей, в частности, что $\left.\operatorname{Im} a^{\mathrm{D}}\right|_{m= \pm \Lambda}=0$.

Однако, чтобы почувствовать разницу между безмассовыми монополями и дионами, следует аккуратно рассмотреть вещественную часть интеграла (21) с учетом логарифмического разреза в $m$-плоскости. Эту вещественную часть можно выбрать равной нулю при $m=\Lambda$, тогда

$$
\left.\operatorname{Re} a^{\mathrm{D}}\right|_{m=\Lambda}=0,\left.\quad \operatorname{Re} a^{\mathrm{D}}\right|_{m=-\Lambda}=2 m=-2 a .
$$

Это означает, что при столкновении кварковой сингулярности $u_{\mathrm{Q}}$ с $u_{\mathrm{M}}$ возникает безмассовый монополь с $\left|a^{\mathrm{D}}\right|=0$, в то время как если $u_{\mathrm{Q}}$ сталкивается с $u_{\mathrm{D}}$, то мы получаем $\left|a^{\mathrm{D}}+2 a\right|=0$, т.е. обращение в нуль массы $(1,1)=[2,1]$-диона. Здесь,

2) Например, уже матрица периодов кривой (13) не является хорошо определенным объектом. Легко проверить, что в точке Аргиреса-Дугласа при $m=\Lambda$ имеем $\tau_{*}=i$, как это и следует из условия самодуальности [3], [14], поскольку кривая (13) вырождается в $y^{2} \sim(x+m)^{3}$, что как раз отвечает данному предельному значению (см., например, справочник [15]). 
следуя работам [6], [2], необходимо особо отметить, что “гомологический” электрический заряд $[2,1]$ этого диона отличается от электрического заряда, принятого в физической нормировке $(1,1)$, в то время как магнитные заряды в обеих нормировках совпадают. Электрический заряд этого диона совпадает с зарядом W-бозона и равен удвоенному заряду кварка: последний равен $(1 / 2,0)$ в физической нормировке, но отвечает одному $A$-циклу (т. е. равен $[1,0])$ в гомологической нормировке, поэтому электрический заряд этого диона (как и $\mathrm{W}$-бозона) отвечает двум $A$-циклам.

В асимптотике для (21) имеем

$$
a^{\mathrm{D}} \underset{m \rightarrow \infty}{\simeq} \frac{i}{\pi} 2 m\left(\ln \frac{2 m}{\Lambda}-1\right)+\cdots .
$$

Для производных выражения (21) по массам получаем

$$
\begin{aligned}
\frac{\partial a^{\mathrm{D}}}{\partial m} & =-\frac{i}{\pi} \ln \frac{m-\sqrt{m^{2}-\Lambda^{2}}}{m+\sqrt{m^{2}-\Lambda^{2}}} \\
\frac{\partial^{2} a^{\mathrm{D}}}{\partial m^{2}} & =\frac{2 i}{\pi \sqrt{m^{2}-\Lambda^{2}}}
\end{aligned}
$$

В точках, где вторая производная сингулярна, можно написать разложения по дробным степеням: при $m=\Lambda$

$$
a^{\mathrm{D}} \underset{m \rightarrow \Lambda}{\simeq} \frac{4 i}{3 \pi} \sqrt{\frac{2}{\Lambda}} \cdot(m-\Lambda)^{3 / 2}+\cdots,
$$

аналогично при $m=-\Lambda$

$$
a^{\mathrm{D}} \underset{m \rightarrow-\Lambda}{\simeq} 2 m+\frac{4}{3 \pi} \sqrt{\frac{2}{\Lambda}} \cdot(m+\Lambda)^{3 / 2}+\cdots .
$$

Эти выражения могут быть легко проанализированы на предмет наличия нетривиальных монодромий в $m$-плоскости.

В частности, из (26) следует, что, будучи чисто мнимым при $t=m-\Lambda>0$, значение периода $a^{\mathrm{D}}$ при движении в область отрицательных значений $t=m-$ $\Lambda<0$ становится либо положительным, либо отрицательным - в зависимости от выбранного пути в $m$-плоскости (знака угловой переменной в $t=\varepsilon e^{ \pm i \varphi}$ при $t \approx 0$ ). Формула (26) утверждает, что

$$
a^{\mathrm{D}} \approx \frac{4 i}{3 \pi} \sqrt{\frac{2}{\Lambda}} t^{3 / 2}= \pm \frac{4}{3 \pi} \sqrt{\frac{2}{\Lambda}} \varepsilon^{3 / 2} .
$$

Предположим, что мы взяли $u=m^{2}+\delta$, причем $m \gg|\delta|>0$, в окрестности кваркового вакуума. Тогда $a=-\sqrt{u} \simeq-m-\delta / 2 m$, другими словами, $a+m \simeq$ $-\delta / 2 m$. Когда $m$ стремится к $\Lambda$ при $t \rightarrow 0$, масса легкого кварка приблизительно равна

$$
|a+m| \simeq\left|\frac{\delta}{2 m}\right| \simeq\left|\frac{\delta}{2 \Lambda}\right| \gtrsim 0
$$


Из формулы (28) следует, что при стремлении к отрицательным $t$ в зависимости от выбранного пути в $m$-плоскости после пересечения критической линии масса одного из дионов с зарядами $(1 / 2, \pm 1)=[1, \pm 1]$ становится меньше, чем масса кварка. Эти условия можно формально записать в виде

$$
\begin{array}{llrl}
\operatorname{sign}(\operatorname{Im} t) & =\operatorname{sign}(\delta): & & \left|a+m+a^{\mathrm{D}}\right|<|a+m|, \\
\operatorname{sign}(\operatorname{Im} t)=-\operatorname{sign}(\delta): & & \left|a+m-a^{\mathrm{D}}\right|<|a+m| .
\end{array}
$$

Если такое случается, скажем, для положительного магнитного заряда, т. е. знака плюс $a^{\mathrm{D}}$ в неравенстве $\left|a+m+a^{\mathrm{D}}\right|<|a+m|$, то кварк может испустить безмассовый антимонополь и превратиться в дион с зарядом $(1 / 2,1)=[1,1]$ с законом сохранения зарядов $[1,0]+[0,1]=[1,1]$. В противоположном случае следует изменить знак заряда монополя, т. е. общая формула имеет вид

$$
[1,0]+[0, q]=[1, q], \quad q= \pm 1,
$$

причем конкретный выбор знака $q$, как мы увидим ниже, физически ненаблюдаем. Похожим образом на другой критической линии, при $m=-\Lambda$, возникает процесс с законом сохранения $[1, q]=[2, q]+[-1,0]$, где опять $q= \pm 1$, или дион с зарядом $[1, q]$ распадается в $[2, q]$-дион, безмассовый при $m=-\Lambda$ в силу $(22)$, и кварк $[-1,0]$, который можно считать “двойственным" [1,0]-кварку после изменения знака массы $m \leftrightarrow-m$ (см. работу [2]).

Проведенный в этом пункте анализ может быть дополнен непосредственным рассмотрением процесса перестановки точек ветвления на кривой Виттена-Зайберга (3) в окрестности сингулярности [2], [18], который приводит в основном к тем же заключениям.

3.2. Теории с $N_{\mathrm{c}}=3$ и $N_{\mathrm{f}}=4,5$. Обратимся теперь непосредственно к теории с группой $S U(3)$ и $N_{\mathrm{f}}=4$ с попарно совпадающими массами $m_{1}=m_{3}$ и $m_{2}=m_{4}$, когда кривая (3) принимает вид

$$
y^{2}=\left(x-\phi_{1}\right)^{2}\left(x-\phi_{2}\right)^{2}\left(x+\phi_{1}+\phi_{2}\right)^{2}-4 \Lambda^{2}\left(x+m_{1}\right)^{2}\left(x+m_{2}\right)^{2} .
$$

Если положить $\phi_{i}=-m_{A} \delta_{i, A}$ при $i, A=1,2$ для выбранного кваркового вакуума, то кривая (32) вырождается в

$$
\begin{aligned}
y^{2} & =\left(x+m_{1}\right)^{2}\left(x+m_{2}\right)^{2}\left(\left(x-m_{1}-m_{2}\right)^{2}-4 \Lambda^{2}\right)= \\
& =\left(x+m_{1}\right)^{2}\left(x+m_{2}\right)^{2}\left((x-M)^{2}-4 \Lambda^{2}\right) \equiv\left(x+m_{1}\right)^{2}\left(x+m_{2}\right)^{2} Y^{2},
\end{aligned}
$$

где $Y^{2}=(x-M)^{2}-4 \Lambda^{2}$ и $M=m_{1}+m_{2}$. Дифференциал Виттена-Зайберга (7) в этом случае принимает вид

$$
d S=\frac{x d x}{Y}+\frac{x d x}{x+m_{1}}+\frac{x d x}{x+m_{2}},
$$

где $p=x-M, Y^{2}=p^{2}-4 \Lambda^{2}$. Его нормировка $(k=1,2)$

$$
\begin{aligned}
& \frac{1}{2 \pi i} \oint_{x=-m_{k}} d S_{+}=\frac{1}{2 \pi i} \oint_{A_{k}^{+}} d S_{+}=a_{k}=-m_{k} \\
& \frac{1}{2 \pi i} \oint_{x=-m_{k}} d S_{-}=\operatorname{res}_{x=-m_{k}} d S_{-}-\frac{1}{2 \pi i} \oint_{A_{k}^{-}} d S_{-}=-2 m_{k}+a_{k}=-m_{k}
\end{aligned}
$$


получается совершенно аналогично (15) для каждой из масс $m_{1,2}$ в (33), (34). Очевидно, что вычеты (35) в точности отвечают обращению в нуль эффективных масс кварков, $a_{k}+m_{k}=0, k=1,2$, в выбранном кварковом вакууме с двумя выпавшими в конденсат ароматами.

Обратимся теперь к двойственным (35) B-периодам, отвечающим вследствие равенств $\boldsymbol{\mu}_{i} \cdot \boldsymbol{\alpha}_{j}=\delta_{i j}, i, j=1,2$, массам монополей. Они опять вычисляются как разности $\left.S_{+}\right|_{x=-m_{k}}-\left.S_{-}\right|_{x=-m_{k}}, k=1,2$, значений абелева интеграла от дифференциала (34),

$$
S=Y+M \ln (x-M+Y)+2 x-m_{1} \ln \left(x+m_{1}\right)-m_{2} \ln \left(x+m_{2}\right) .
$$

Поскольку эти выражения также сингулярны при $x+m_{k}=0$, следует ввести регуляторы $\epsilon_{k}^{ \pm}, k=1,2$, на каждом из листов кривой (33). Как и в (19), эти регуляторы определяются из следующих соотношений $(k=1,2)$ :

$$
\left.\phi_{+}\right|_{x=-m_{k}}-\left.\phi_{-}\right|_{x=-m_{k}}=\ln \frac{\epsilon_{k}^{+}}{\epsilon_{k}^{-}}+\ln \frac{M+m_{k}-\sqrt{\left(M+m_{k}\right)^{2}-4 \Lambda^{2}}}{M+m_{k}+\sqrt{\left(M+m_{k}\right)^{2}-4 \Lambda^{2}}}=0,
$$

где

$$
\phi=\int\left(\frac{d x}{Y}+\frac{d x}{x+m_{1}}+\frac{d x}{x+m_{2}}\right)=\ln (x-M+Y)+\ln \left(x+m_{1}\right)+\ln \left(x+m_{2}\right) .
$$

Поэтому

$$
\begin{aligned}
& \left.S_{+}\right|_{x=-m_{k}}-\left.S_{-}\right|_{x=-m_{k}}=-m_{k} \ln \frac{\epsilon_{k}^{+}}{\epsilon_{k}^{-}}+\left.2 Y\right|_{x=-m_{k}}+M \ln \frac{-m_{k}-M+\left.Y\right|_{x=-m_{k}}}{-m_{k}-M-\left.Y\right|_{x=-m_{k}}}= \\
& =2 \sqrt{\left(m_{k}+M\right)^{2}-4 \Lambda^{2}}+\left(m_{k}+M\right) \ln \frac{m_{k}+M-\sqrt{\left(m_{k}+M\right)^{2}-4 \Lambda^{2}}}{m_{k}+M+\sqrt{\left(m_{k}+M\right)^{2}-4 \Lambda^{2}}} .
\end{aligned}
$$

Таким образом (ср. с формулой (21)),

$$
\begin{aligned}
a_{1}^{\mathrm{D}}= & \frac{1}{2 \pi i} \oint_{B_{1}} d S=-\frac{i}{\pi}\left(\sqrt{\left(2 m_{1}+m_{2}\right)^{2}-4 \Lambda^{2}}+\right. \\
& \left.+\left(m_{1}+\frac{m_{2}}{2}\right) \ln \frac{2 m_{1}+m_{2}-\sqrt{\left(2 m_{1}+m_{2}\right)^{2}-4 \Lambda^{2}}}{2 m_{1}+m_{2}+\sqrt{\left(2 m_{1}+m_{2}\right)^{2}-4 \Lambda^{2}}}\right)
\end{aligned}
$$

и

$$
\begin{aligned}
a_{2}^{\mathrm{D}}= & \frac{1}{2 \pi i} \oint_{B_{2}} d S=-\frac{i}{\pi}\left(\sqrt{\left(m_{1}+2 m_{2}\right)^{2}-4 \Lambda^{2}}+\right. \\
& \left.+\left(\frac{m_{1}}{2}+m_{2}\right) \ln \frac{m_{1}+2 m_{2}-\sqrt{\left(m_{1}+2 m_{2}\right)^{2}-4 \Lambda^{2}}}{m_{1}+2 m_{2}+\sqrt{\left(m_{1}+2 m_{2}\right)^{2}-4 \Lambda^{2}}}\right) .
\end{aligned}
$$

Полученные выражения, очевидно, обладают нужными свойствами, как и в случае теории с $N_{\mathrm{c}}=2$ и $N_{\mathrm{f}}=2$, которая эффективно описывает динамику в подгруппах калибровочной группы $S U(3)$, отвечающих корням $\boldsymbol{\alpha}_{1,2}$. 
В теории с $N_{\mathrm{f}}=5$ вычисление совершенно аналогично, хотя сами конечные формулы имеют несколько более сложный вид. Вырожденную кривую можно опять записать в форме

$$
y^{2}=\left(x+m_{1}\right)^{2}\left(x+m_{2}\right)^{2} Y^{2},
$$

где теперь

$$
Y^{2}=p^{2}-4 \Lambda\left(x+m_{5}\right), \quad p=x-M+\Lambda=x-m_{1}-m_{2}+\Lambda .
$$

Она оснащена дифференциалом Виттена-Зайберга (7), имеющим в данном случае вид

$$
d S=\frac{x d x}{Y}-\frac{x p}{2 Y} \frac{d x}{x+m_{5}}+\frac{1}{2} \frac{x d x}{x+m_{5}}+\frac{x d x}{x+m_{1}}+\frac{x d x}{x+m_{2}} .
$$

Вычисление $B$-периодов дифференциала (43) аналогично случаю $N_{\mathrm{f}}=4$ приводит к результату

$$
\begin{aligned}
& a_{k}^{\mathrm{D}}= \frac{1}{2 \pi i} \oint_{B_{k}} d S=-\frac{i}{\pi}\left(Y_{k}+\left(M+\frac{m_{k}+m_{5}}{2}\right) \ln \frac{M+m_{k}+\Lambda-Y_{k}}{M+m_{k}+\Lambda+Y_{k}}+\right. \\
&\left.+\frac{m_{k}-m_{5}}{2} \ln \frac{a+b_{+} m_{k}-b_{-} Y_{k}}{a+b_{+} m_{k}+b_{-} Y_{k}}\right), \quad k=1,2,
\end{aligned}
$$

где $a=(M-\Lambda)^{2}+m_{5}(M-3 \Lambda), b_{ \pm}=M+m_{5} \pm \Lambda$, с двумя параболами $Y_{1,2}$ в $\left(m_{1}, m_{2}\right)$-плоскости, определенными уравнениями

$$
\begin{aligned}
& Y_{1}^{2}=\left(2 m_{1}+m_{2}\right)^{2}-\Lambda\left(2 m_{5}+m_{2}\right)+\Lambda^{2}=0, \\
& Y_{2}^{2}=\left(m_{1}+2 m_{2}\right)^{2}-\Lambda\left(2 m_{5}+m_{1}\right)+\Lambda^{2}=0 .
\end{aligned}
$$

Эти параболы, естественным образом возникающие в процессе вычисления периодов, играют роль границ деформированной области режима сильной связи в случае теории с $N_{\mathrm{f}}=5$, если сравнивать ее с теорией при $N_{\mathrm{f}}=4$, в которой эти границы представляют собой прямые $m_{1}+2 m_{2}= \pm 2 \Lambda$ и $2 m_{1}+m_{2}= \pm 2 \Lambda$.

Легко проверить, что периоды (44) обладают всеми необходимыми свойствами. Заметим также, что представленный в настоящей работе вывод соотношений (44) не имеет ничего общего с содержащимися в работах [17], [18] непрямыми методами, которые основаны на параллелях между двумерным и четырехмерным анализом проблемы.

Формулы (40), (41) и (44) немедленно приводят к простым физическим следствиям [2]. Обращение в нуль величин $a_{k}^{\mathrm{D}}=\boldsymbol{\alpha}_{k} \cdot \mathbf{a}^{\mathrm{D}}$ отвечает безмассовости монополей с зарядами

$$
M_{k}=\sqrt{2}\left(\mathbf{n}_{\mathrm{e}} \oplus \mathbf{n}_{\mathrm{m}}\right)_{k}^{M}=\mathbf{0} \oplus \boldsymbol{\alpha}_{k}, \quad k=1,2,
$$

в терминах корней калибровочной группы $S U(3)$ (см. рис. 1). Для периода (40) это происходит при $2 m_{1}+m_{2}=2 \Lambda$, а для периода (41) - при $m_{1}+2 m_{2}=2 \Lambda$.

Очевидно, что $\operatorname{Im} a_{1}^{\mathrm{D}}=0$ также при $2 m_{1}+m_{2}=-2 \Lambda$, и аналогично $\operatorname{Im} a_{2}^{\mathrm{D}}=0$, если $m_{1}+2 m_{2}=-2 \Lambda$. Однако вещественная часть выражения (40) при $2 m_{1}+m_{2}=$ 


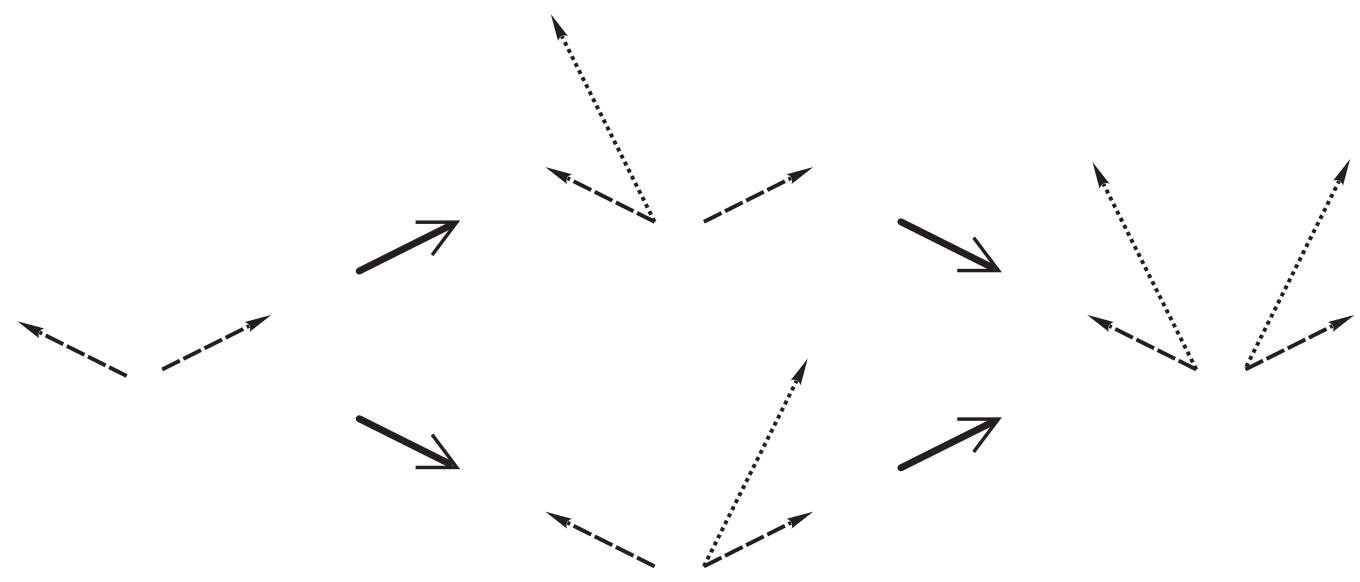

Рис. 3. Изменение квантовых чисел, диктуемое монодромиями: дублет кварков (в слабой связи) превращается в дублет дионов (в сильной связи) в зависимости от выбора того или иного пути в массовой плоскости.

$-2 \Lambda$ равна $2 m_{1}+m_{2}=-\boldsymbol{\alpha}_{1} \cdot \mathbf{a}-$ массе $\mathrm{W}$-бозона с зарядом $\boldsymbol{\alpha}_{1}$. Тем самым при $2 m_{1}+m_{2}=-2 \Lambda$ мы получаем безмассовый дион с зарядом

$$
D_{1}=\sqrt{2}\left(\mathbf{n}_{\mathrm{e}} \oplus \mathbf{n}_{\mathrm{m}}\right)_{1}^{\mathrm{D}}=\boldsymbol{\alpha}_{1} \oplus \boldsymbol{\alpha}_{1}
$$

и аналогично - безмассовый дион с зарядом

$$
D_{2}=\sqrt{2}\left(\mathbf{n}_{\mathrm{e}} \oplus \mathbf{n}_{\mathrm{m}}\right)_{2}^{\mathrm{D}}=\boldsymbol{\alpha}_{2} \oplus \boldsymbol{\alpha}_{2}
$$

при $m_{1}+2 m_{2}=-2 \Lambda$. Формулы (44) показывают, что при $Y_{k}=0, k=1,2$ (т.е. на каждой из парабол $(45)$ в $\left(m_{1}, m_{2}\right)$-плоскости), мнимая часть соответствующего периода $\operatorname{Im} a_{k}^{\mathrm{D}}$ равна нулю, в то время как вещественная часть $\operatorname{Re} a_{k}^{\mathrm{D}}$ испытывает скачок при переходе с положительной на отрицательную ветвь соответствующей $k$-й параболы.

Анализ вычисленных периодов (40), (41) и (44) приводит к следующей картине изменения квантовых чисел благодаря монодромиям (см. рис. 3): начав с конденсата двух кварков в слабой связи, мы приходим в фазе сильной связи к конденсату из двух легких дионов. Формула (31) предыдущего пункта описывает проекцию рис. 3 на горизонтальную ось. В терминах весовых и корневых векторов из рис. 1 мы обнаруживаем, что каждый кварк с "весоподобным" электрическим зарядом приобретает также “корнеподобный” магнитный заряд и превращается в дион, природа которого отлична от природы дионов (47) и (48): в фазе сильной связи мы получаем конденсат легких дионов $\Psi_{1,2}$ с зарядами ${ }^{3)}$

$$
\mathcal{D}_{k}=\boldsymbol{\mu}_{k} \oplus \boldsymbol{\alpha}_{k}=\sqrt{2}\left(\mathbf{n}_{\mathrm{e}} \oplus \mathbf{n}_{\mathrm{m}}\right)_{k}^{\mathcal{D}} \equiv \sqrt{2} \mathbf{n}^{k}, \quad k=1,2,
$$

вместо конденсата кварков [2].

\footnotetext{
3) Отметим сразу, что каллиграфической буквой $\mathcal{D}$ мы обозначаем дионы с "весоподобными" электрическими зарядами, чтобы не путать их с дионными родственниками монополей, заряды которых (47) и (48) задаются исключительно корневыми векторами.
} 


\section{4. КОНФАЙНМЕНТ}

Поскольку в режиме слабой связи изначальной теории при больших $m$ в $r=2$ вакууме кварки находятся в хиггсовской фазе, объектами конфайнмента являются монополи. Два из трех элементарных $S U(3)$-монополя с магнитными зарядами $\boldsymbol{\alpha}_{1,2}$ (см. формулы (46)) приклеиваются к концам элементарных струн, в то время как третий, с зарядом $\boldsymbol{\alpha}_{12}=\boldsymbol{\alpha}_{1}-\boldsymbol{\alpha}_{2}$, реализуется как узел соприкосновения двух элементарных струн [1]. В области сильной связи эти аргументы можно воспроизвести для дуальной теории легких дионов (49), построив фундаментальные струны из лагранжиана эффективной теории $\mathcal{L} \sim \sum_{k=1,2}\left|\nabla \Psi_{k}\right|^{2}+\cdots$, в котором минимальное взаимодействие

$$
\nabla \Psi_{k}=\left(\partial-i \mathcal{D}_{k} \cdot\left(\mathbf{A} \oplus \mathbf{A}^{\mathrm{D}}\right)\right) \Psi_{k}=\left(\partial-i\left(\boldsymbol{\mu}_{k} \cdot \mathbf{A}+\boldsymbol{\alpha}_{k} \cdot \mathbf{A}^{\mathrm{D}}\right)\right) \Psi_{k}
$$

определяется дионными зарядами (49).

Элементарные заряды $\mathbb{Z}_{2}$-струн $S_{1,2}$ фиксируются зарядами дионов (49) путем анализа поведения калибровочных потенциалов на пространственной бесконечности:

$$
S_{i}: \quad \mathcal{D}_{k} \cdot\left(\mathbf{A} \oplus \mathbf{A}^{\mathrm{D}}\right)=\boldsymbol{\mu}_{i} \cdot \mathbf{A}+\boldsymbol{\alpha}_{i} \cdot \mathbf{A}^{\mathrm{D}} \sim \delta_{i k} d \theta, \quad i, k=1,2,
$$

где $\theta$ обозначает угол в плоскости, перпендикулярной направлению струны. Отсюда следует, что для декартовых проекций $A_{3} \sim \boldsymbol{\alpha}_{12} \cdot \mathbf{A} \sim\left(\boldsymbol{\mu}_{1}-\boldsymbol{\mu}_{2}\right) \cdot \mathbf{A}$ и $A_{8} \sim\left(\boldsymbol{\alpha}_{1}+\right.$ $\left.\boldsymbol{\alpha}_{2}\right) \cdot \mathbf{A} \sim-\boldsymbol{\mu}_{12} \cdot \mathbf{A}$ (отвечающих горизонтальному и вертикальному направлениям на рис. 1) с индексами 3,8 , унаследованными от диагональных матриц Гелл-Манна, мы имеем

$$
A_{3}+A_{3}^{\mathrm{D}} \sim d \theta, \quad \frac{A_{8}}{\sqrt{3}}+\sqrt{3} A_{8}^{\mathrm{D}} \sim d \theta .
$$

Ортогональные к (52) комбинации потенциалов стремятся на бесконечности к нулю:

$$
A_{3}-A_{3}^{\mathrm{D}} \sim 0, \quad A_{8}^{\mathrm{D}}-3 A_{8} \sim 0 .
$$

В результате получаем [2] для зарядов (51) в терминах потоков ${ }^{4)}$

$$
\begin{aligned}
\oint d x \cdot\left(\mathbf{A}^{\mathrm{D}} \oplus \mathbf{A}\right) & =\oint d x \cdot\left(A_{3}^{\mathrm{D}}, A_{3} ; A_{8}^{\mathrm{D}}, A_{8}\right)= \\
& =4 \pi\left(-n_{3}^{\mathrm{e}}, n_{3}^{\mathrm{m}} ;-n_{8}^{\mathrm{e}}, n_{8}^{\mathrm{m}}\right)=4 \pi\left(-\mathbf{n}^{\mathrm{e}} \oplus \mathbf{n}^{\mathrm{m}}\right),
\end{aligned}
$$

что заряд $S_{1}$-струны равен

$$
\mathbf{n}_{S_{1}}=\left(-\frac{1}{4}, \frac{1}{4} ;-\frac{3 \sqrt{3}}{20}, \frac{\sqrt{3}}{20}\right) \equiv \frac{S_{1}}{\sqrt{2}},
$$

в то время как заряд $S_{2}$-струны, возникающей из намоток на пространственной бесконечности фазы второго диона, равен

$$
\mathbf{n}_{S_{2}}=\left(\frac{1}{4},-\frac{1}{4} ;-\frac{3 \sqrt{3}}{20}, \frac{\sqrt{3}}{20}\right) \equiv \frac{S_{2}}{\sqrt{2}} .
$$

\footnotetext{
4) Такое определение обеспечивает равенство заряда струны и заряда пробного диона, который приклеивается к ее концу (не обязательно при этом существуя в спектре самой теории).
} 
Теперь легко проверить, что для каждого из трех $S U(3)$-монополей действительно наблюдается конфайнмент, вызванный наличием этих струн [2]. Для монополей с зарядами $\mathbf{0} \oplus \boldsymbol{\alpha}_{1,2}$ или $(0, \pm 1 / 2 ; 0, \sqrt{3} / 2)$ можно написать следующие разложения:

$$
\begin{aligned}
& \frac{1}{\sqrt{2}}\left(\mathbf{0} \oplus \boldsymbol{\alpha}_{1}\right)=\left(0, \frac{1}{2} ; 0, \frac{\sqrt{3}}{2}\right)=\mathbf{n}_{S_{1}}+\frac{7}{10} \mathbf{n}^{1}+\frac{2}{10} \mathbf{n}^{2}, \\
& \frac{1}{\sqrt{2}}\left(\mathbf{0} \oplus \boldsymbol{\alpha}_{2}\right)=\left(0,-\frac{1}{2} ; 0, \frac{\sqrt{3}}{2}\right)=\mathbf{n}_{S_{2}}+\frac{2}{10} \mathbf{n}^{1}+\frac{7}{10} \mathbf{n}^{2},
\end{aligned}
$$

где $\mathbf{n}^{k}=\mathcal{D}_{k} / \sqrt{2}, k=1,2$, представляют собой нормированные заряды дионов (49). Соотношения (57) показывают, что только часть монополь-антимонопольного потока "запирается" внутри мировой поверхности струны, в то время как оставшаяся часть просто экранируется дионным конденсатом.

Для третьего $\boldsymbol{\alpha}_{12}$-монополя в $S U(3)$-случае формулы приобретают более элегантный вид: из (57) получаем, что

$$
\frac{1}{\sqrt{2}}\left(\mathbf{0} \oplus \boldsymbol{\alpha}_{12}\right)=(0,1 ; 0,0)=\mathbf{n}_{S_{1}}-\mathbf{n}_{S_{2}}+\frac{1}{2}\left(\mathbf{n}^{1}-\mathbf{n}^{2}\right),
$$

откуда следует, что этот монополь также находится в фазе конфайнмента, представляя собой узел стыковки двух элементарных струн $S_{1}$ и $S_{2}$. Динамику в неабелевом направлении $\boldsymbol{\alpha}_{12}$ можно изобразить в виде "разностного диона" $\mathcal{D}_{1}-\mathcal{D}_{2}=$

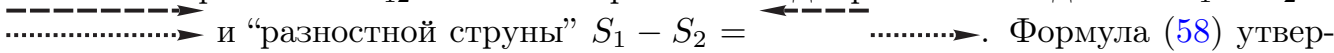
ждает, что вследствие полного экранирования электрического заряда "разностной

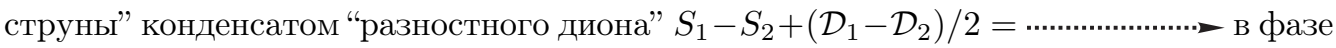
конфайнмента находятся лишь магнитные заряды $(S U(2))$-монополя.

Вернемся теперь к проблеме неоднозначности. Заметим, что при переходе в область сильной связи знак магнитного заряда, приобретаемый легким кварком при превращении в легкий дион, является ненаблюдаемым, поскольку зависит от выбора конкретной траектории в массовом пространстве (см. обсуждение в п. 3.1). Это означает, что вместо эффективной теории легких дионов с зарядами (49) может также возникнуть теория, в которой заряды дионов равны

$$
\sqrt{2} \tilde{\mathbf{n}}^{1}=\boldsymbol{\mu}_{1} \oplus\left(-\boldsymbol{\alpha}_{1}\right), \quad \sqrt{2} \tilde{\mathbf{n}}^{2}=\boldsymbol{\mu}_{2} \oplus\left(-\boldsymbol{\alpha}_{2}\right) .
$$

Эта теория на самом деле эквивалентна той, которую мы только что обсудили: единственное различие между ними заключается в изменении на противоположный знаков компонент дуального калибровочного поля $A^{\mathrm{D}}$. В результате вместо $(55)$ и (56) для зарядов элементарных струн мы будем иметь выражения

$$
\tilde{\mathbf{n}}_{S_{1}}=\left(\frac{1}{4}, \frac{1}{4} ; \frac{3 \sqrt{3}}{20}, \frac{\sqrt{3}}{20}\right), \quad \tilde{\mathbf{n}}_{S_{2}}=\left(-\frac{1}{4},-\frac{1}{4} ; \frac{3 \sqrt{3}}{20}, \frac{\sqrt{3}}{20}\right)
$$


т. е. электрические компоненты зарядов окажутся противоположными по знаку. Легко, однако, проверить, что вместо (57) теперь можно написать разложение

$$
\begin{aligned}
& \frac{1}{\sqrt{2}}\left(\mathbf{0} \oplus \boldsymbol{\alpha}_{1}\right)=\left(0, \frac{1}{2} ; 0, \frac{\sqrt{3}}{2}\right)=\tilde{\mathbf{n}}_{S_{1}}-\frac{7}{10} \tilde{\mathbf{n}}^{1}-\frac{2}{10} \tilde{\mathbf{n}}^{2}, \\
& \frac{1}{\sqrt{2}}\left(\mathbf{0} \oplus \boldsymbol{\alpha}_{2}\right)=\left(0,-\frac{1}{2} ; 0, \frac{\sqrt{3}}{2}\right)=\tilde{\mathbf{n}}_{S_{2}}-\frac{2}{10} \tilde{\mathbf{n}}^{1}-\frac{7}{10} \tilde{\mathbf{n}}^{2},
\end{aligned}
$$

и мы получим, что основной вывод работы [2] остается неизменным при замене знаков магнитных компонент зарядов дионов: объектами, испытывающими конфайнмент благодаря струнам в эффективной теории, остаются монополи, причем часть струнного заряда экранируется дионным конденсатом. Это означает, в частности, что в зависимости от выбора конкретного пути в массовой плоскости при переходе к фазе сильной связи изначальной теории можно получить различные заряды дионов и струнных решений в эффективной теории. Однако эта неоднозначность не влияет на наблюдаемые физические результаты: в любом случае будет наблюдаться конфайнмент тех же самых магнитных монополей.

\section{5. ЗАКЛЮЧЕНИЕ}

Мы обсудили некоторые особенности теории Виттена-Зайберга для вырожденных кривых в окрестности $\mathcal{N}=1$ вакуумов суперсимметричной КХД. Вычисление периодов для этих сингулярных кривых позволяет понять, как именно изменяются квантовые числа легких состояний при переходе из области слабой связи в область сильной связи по какой-либо траектории в пространстве модулей масс теории. Этот анализ позволяет получить точную картину конфайнмента в суперсимметричной КХД в сильной связи.

Показано, что вычисление периодов может быть проведено непосредственно с использованием техники интегрируемых систем. В данном контексте это просто означает, что точное решение суперсимметричной калибровочной теории характеризуется кривой с двумя мероморфными дифференциалами, периоды которых фиксированы. Фиксации периодов этих дифференциалов оказывается достаточно, чтобы обеспечить регуляризацию сингулярной кривой, отвечающей $\mathcal{N}=1$ вакууму, и поэтому массы легких состояний вычисляются точно. Это является еще одним нетривиальным применением классических интегрируемых систем в квантовой теории.

Благодарности. Работа выполнена при частичной поддержке Российского федерального агенства по атомной энергии, Программы поддержки ведущих научных школ (грант НШ-1615.2008.2), РФФИ (гранты № 08-01-00667, 09-02-90493-Укр, 09-02-93105-CNRSL, 09-01-92440-CE) и университета города Киото, а также Министерством образования и науки РФ (контракт 02.740.11.0608). Автор благодарен Институту теоретической физики им. Юкавы, где была выполнена основная часть настоящей работы, за гостеприимство.

\section{Список литературы}

[1] A. Marshakov, A. Yung, Nucl. Phys. B, 647:1-2 (2002), 3-48, arXiv: hep-th/0202172.

[2] A. Marshakov, A. Yung, Nucl. Phys. B, 831:1-2 (2010), 72-104, arXiv: 0912.1366. 
[3] A. Bilal, F. Ferrari, Nucl. Phys. B, 516:1-2 (1998), 175-228, arXiv: hep-th/9706145.

[4] A. Ritz, A. Vainshtein, Nucl. Phys. B, 617:1-3 (2001), 43-70, arXiv: hep-th/0102121.

[5] N. Seiberg, E. Witten, Nucl. Phys. B, 426:1 (1994), 19-52; Erratum, 430 (1994), 485-486, arXiv: hep-th/9407087.

[6] N. Seiberg, E. Witten, Nucl. Phys. B, 431:3 (1994), 484-550, arXiv: hep-th/9408099.

[7] A. Hanany, Y. Oz, Nucl. Phys. B, 452:1-2 (1995), 283-312, arXiv: hep-th/9505075.

[8] P. C. Argyres, M. R. Plesser, N. Seiberg, Nucl. Phys. B, 471:1-2 (1996), 159-194, arXiv: hep-th/9603042.

[9] A. Gorsky, A. Marshakov, A. Mironov, A. Morozov, Phys. Lett. B, 380:1-2 (1996), 75-80, arXiv: hep-th/9603140.

[10] D. Gaiotto, $N=2$ dualities, arXiv: 0904.2715.

[11] T. Eguchi, K. Maruyoshi, JHEP, 2010, 022, 21 pp., arXiv: 0911.4797.

[12] A. Losev, A. Marshakov, N. Nekrasov, "Small instantons, little strings and free fermions", From Fields to Strings: Circumnavigating Theoretical Physics, Ian Kogan memorial collection. V.1, eds. M. Shifman, A. Vainshtein, J. Wheater, World Sci., Singapore, 2005, 581-621, arXiv: hep-th/0302191.

[13] A. Marshakov, N.A. Nekrasov, JHEP, 01 (2007), 104, 39 pp., arXiv: hep-th/0612019; A. В. Маршаков, ТMФ, 154:3 (2008), 424-450, arXiv: 0706.2857.

[14] P. C. Argyres, M. R. Douglas, Nucl. Phys. B, 448:1-2 (1995), 93-126, arXiv: hep-th/9505062; P.C. Argyres, M.R. Plesser, N. Seiberg, E. Witten, Nucl. Phys. B, 461:1-2 (1996), 71-84, arXiv: hep-th/9511154; T. Eguchi, K. Hori, K. Ito, S.-K. Yang, Nucl. Phys. B, 471:3 (1996), 430-442, arXiv: hep-th/9603002.

[15] Г. Бейтмен, А. Эрдейи, Высшие трансиендентные функиии. Т. 3: Эллиптические и автоморфные функиии. Функиии Ламе и Матъе, Справочная математическая библиотека, Наука, М., 1967.

[16] I. M. Krichever, Comm. Pure. Appl. Math., 47:4 (1992), 437-475, arXiv: hep-th/9205110.

[17] N. Dorey, JHEP, 11 (1998), 005, 50 pp., arXiv: hep-th/9806056; N. Dorey, T. Hollowood, D. Tong, JHEP, 05 (1999), 006, arXiv: hep-th/9902134.

[18] M. Shifman, A. Yung, Phys.Rev. D, 79:12 (2009), 125012, 23 pp., arXiv: 0904.1035. 